\title{
Herpes Zoster Ophthalmicus and Lateral Rectus Palsy in an Elderly Patient
}

\author{
Ozgur Yalcinbayir Meral Yildiz Gamze Ucan Gunduz \\ Oner Gelisken
}

Department of Ophthalmology, Uludağ University School of Medicine, Bursa, Turkey

\section{Key Words}

Abduction · Cranial nerve palsy · Esotropia · Herpes zoster ophthalmicus · Neuroophthalmology $\cdot$ Ocular motility

\begin{abstract}
Acquired palsy of the lateral rectus presents with horizontal diplopia and has a broad differential. Herpes zoster ophthalmicus- $(\mathrm{HZO})$ related cranial nerve palsy is a transient and self-limiting condition. Systemic antiviral treatment is administered in order to prevent sightthreatening complications. In suspected cases, zosteriform rash should be questioned. One should keep in mind that acquired esotropia in the elderly may sometimes present following
\end{abstract} HZO.

\section{Introduction}

Isolated cranial nerve (CN) VI palsy is relatively common in the elderly population, in contrast to the pediatric population [1]. Most CN VI palsies in the elderly are attributable to microvascular ischemic disease, mainly diabetes mellitus or arterial hypertension. Several other causes, including high intracranial pressure, giant cell arteritis, cavernous sinus mass and multiple sclerosis have also been reported [2].

Herpes zoster is a rare but well-known cause of CN VI palsy. Herpes zoster ophthalmicus (HZO) is the involvement of the ophthalmic division (V1) of the trigeminal nerve and represents approximately $10-20 \%$ of all cases of herpes zoster [3, 4]. The acute course of HZO is usually benign; however, serious ocular complications have been documented [4,5]. Herein, we report a case of CN VI palsy which presented within a week of onset of a zosteriform rash. 


\section{Case Report}

A 73-year-old white male presented to our Emergency Department with complaints of severe leftsided headache, diplopia, and limitation of abduction in the left eye. One week earlier he had developed multiple painful vesiculobullous eruptions on the left side of his forehead and tip of the nose.

On presentation, dark pigmented and crusted patches were scattered in the patient's left trigeminal nerve V1 distribution. His medical history was significant for diabetes mellitus and hypertension. Both systemic examination and blood pressure assessments were completely normal. Laboratory tests revealed that his diabetes was well controlled. There were no specific findings on the neurological examination. Lumbar puncture showed no evidence of meningitis or encephalitis. Polymerase chain reaction testing of the skin lesions and viral culture were deemed unnecessary. Diagnostic workup, for an underlying primary or acquired immunodeficiency, turned out to be negative.

At our initial examination, the patient's visual acuity was 20/20 in the right eye and 20/30 in the left eye. There was no ptosis or anisocoria in the left eye and Hertel's exophthalmometry was normal. The left eye had a mild esotropia of 8 prism diopters at primary gaze. Extraocular muscle movements were painless with restricted abduction of the left eye upon left gaze, compatible with a left CN VI palsy (fig. 1). Temporal arteries were non-palpable. Slit-lamp examination and fundoscopy were normal in both eyes, except for mild conjunctival injection. The intraocular pressure was $14 \mathrm{~mm} \mathrm{Hg}$ bilaterally. Laboratory evaluation results, including erythrocyte sedimentation rate, were all within normal limits. Diffusion-weighted MRI and FLAIR sequences of the orbit and cranium were normal. There was no evidence of acute microvascular ischemic disease. Pre- and post-contrast enhancement $\mathrm{T}_{1}$ images of the orbit and sellae provided no additional clues as to the cause of the ophthalmoplegia.

A diagnosis of CN VI palsy due to HZO was made, and the patient was started on oral valacyclovir $3,000 \mathrm{mg} /$ day for a week. The skin lesions resolved within 2 weeks and a gradual improvement in ocular motility was noted over the following months. In the 4th month of follow-up, the patient presented with a complete recovery of CN VI function with a minimal residual abduction deficit (fig. 2). At that time, his vision was 20/20 in both eyes. During follow-up, the patient had no posttherapeutic neuralgia and suffered no major side effects due to the treatment.

\section{Discussion}

The long intracranial course of CN VI and its fragile nature makes it highly susceptible to inflammation and injury. Involvement of CN VI is the most common form of all CN palsies (43.8\%), followed by CN III (28\%) and CN IV palsies (15\%) [6].

The etiology of CN VI palsy varies by age. In childhood, the leading causes of CN VI palsy are neoplasms; however, in adulthood, vasculopathic disorders are the most common causes. Inflammatory and infectious diseases can be listed as rare incidents among the etiological factors.

In our case, an elderly patient presented one week after the onset of vesiculobullous eruptions with acquired diplopia and mild esotropia. Due to a zosteriform rash and the CN VI palsy, the patient was diagnosed with HZO. Four months after the initiation of antiviral treatment, his CN VI palsy improved dramatically and diplopia resolved.

HZO typically occurs after the reactivation of a latent varicella zoster virus. Extraocular muscle palsies have been reported in $31 \%$ of all HZO cases [7]. The mechanism of ocular motor involvement is still unclear. Several hypotheses including vasculitis, muscle ischemia, contiguous intracavernous radiculomeningitis or cranial motor neuropathy have been postulated. Presumably, the varicella zoster virus may be 
adopting more than one of these mechanisms. The order of $\mathrm{CN}$ involvement is quite different in HZO. The most common palsy in HZO involves CN III. This is followed by CN VI and CN IV palsies, respectively [8].

The treatment of HZO-related CN palsy is controversial. There is an ongoing debate regarding the importance of systemic antiviral treatment and corticosteroids $[4,9,10]$. Systemic antivirals have been reported to reduce the risk of viral dissemination. Besides, their beneficial effects in reducing the clinical signs of HZO have been demonstrated. Therefore, we believe that antiviral therapy should be given in all HZO cases in order to minimize the frequency of potentially sight-threatening complications. On the other hand, some authors have recommended systemic corticosteroids to treat the possible vasculitic component and/or to prevent postherpetic neuralgia [9]. It is well known that steroids should not be given alone (without antiviral therapy) due to concerns about promotion of viral replication. Additionally, they may increase the risk of secondary skin infection [10]. In our case, the patient responded quite well to valacyclovir treatment and had no side effects. Since the patient was already diabetic, we refrained from steroid therapy.

In conclusion, $\mathrm{HZO}$ can present with a wide range of acute and chronic complications. One should keep in mind that, especially in the elderly, it may present with CN palsy and subsequent morbidity. In addition to the more widely known ocular complications, recognition and management of $\mathrm{CN}$ palsies is of importance. In such a case, prompt treatment and close follow-up may provide an opportunity to suppress viral proliferation and persistence in the early stages of the disease. A detailed history and thorough examination of the skin is essential in $\mathrm{CN}$ palsies.

\section{Disclosure Statement}

The authors have no proprietary interests in this study. 


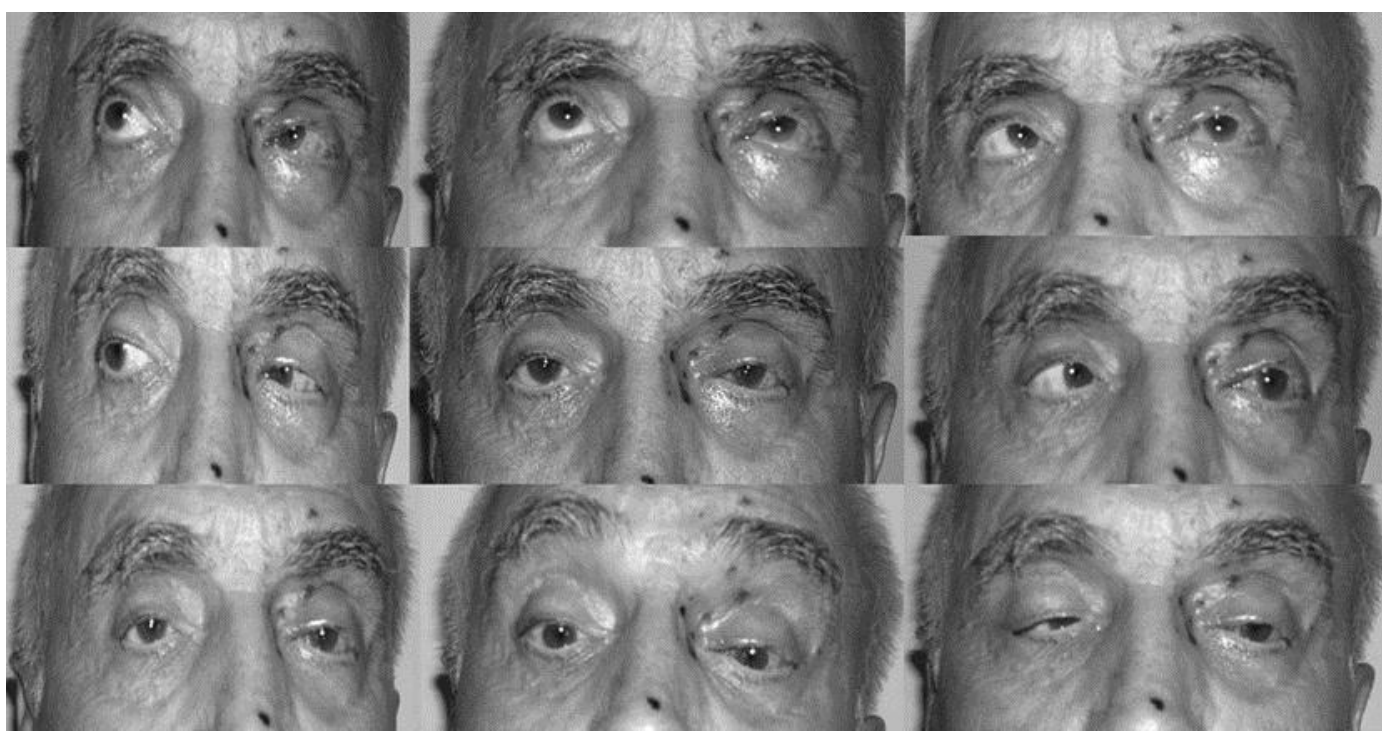

Fig. 1. External photographs of primary gaze (center photo) and 9 cardinal directions of gaze.
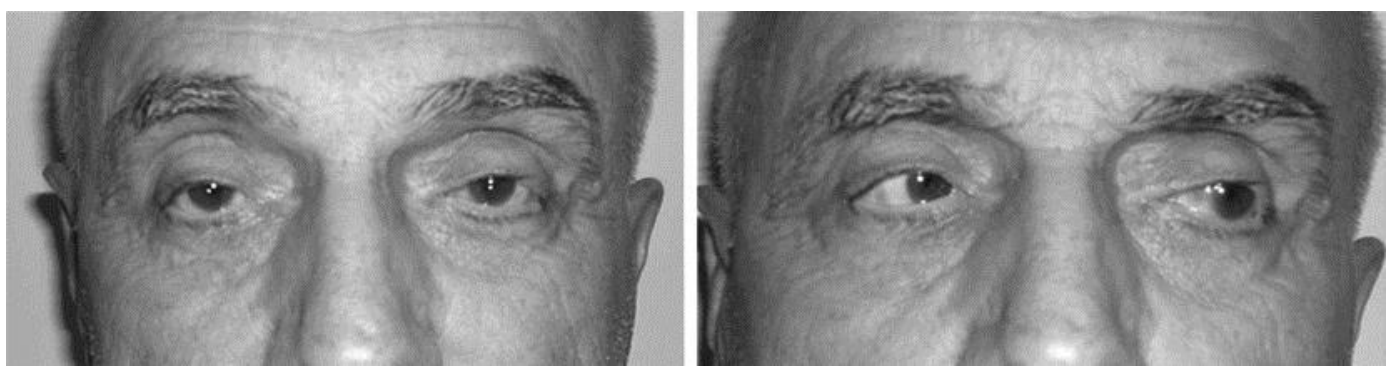

Fig. 2. Recovery of CN VI with minimal abduction deficit in the 4th month of follow-up. 


\section{References}

$>1$ Lee MS, Galetta SL, Volpe NJ, Liu GT: Sixth nerve palsies in children. Pediatr Neurol 1999;20:49-52.

-2 Patel SV, Mutyala S, Leske DA, Hodge DO, Holmes JM: Incidence, associations, and evaluation of sixth nerve palsy using a population-based method. Ophthalmology 2004;111:369-375.

-3 Ragozzino MW: Population-based study of herpes zoster and its sequelae. Medicine (Baltimore) 1982;61:310-316.

4 Liesegang TJ: Herpes zoster ophthalmicus: natural history, risk factors, clinical presentation, and morbidity. Ophthalmology 2008;115:3-12.

-5 Yalcinbayir 0, Gelisken 0, Yilmaz E: Unilateral optic neuritis in a case of herpes zoster ophthalmicus. Neuro-Ophthalmology 2009;33:339-342.

-6 Richards BW, Jones FR Jr, Younge BR: Causes and prognosis in 4,278 cases of paralysis of the oculomotor, trochlear, and abducens cranial nerves. Am J Ophthalmol 1992;113:489-496.

7 Marsh R, Dulley B, Kelly V: External ocular motor palsies in ophthalmic zoster. Br J Ophthalmol 1977;61:677-682.

8 Shin MK, Choi CP, Lee MH: A case of herpes zoster with abducens palsy. J Korean Med Sci 2007;22:905907.

-9 Das D, Pal S, Gangopadhyay PK: Isolated sixth cranial nerve palsy in herpes zoster ophthalmicus. Ann Neurosci 2006;13:92.

10 Whitley RJ, Weiss H, Gnann JW Jr, Tyring S, Mertz GJ, Pappas PG, et al: Acyclovir with and without prednisone for the treatment of herpes zoster. A randomized, placebo-controlled trial. The National Institute of Allergy and Infectious Diseases Collaborative Antiviral Study Group. Ann Intern Med 1996;125:376-383. 